\title{
An unusual presentation of subfrontal meningioma: a case report and literature review for Foster Kennedy syndrome
}

\author{
Shahram Lotfipour $\cdot$ Kris Chiles · J. Akiva Kahn • \\ Tareg Bey $\cdot$ Scott Rudkin
}

Received: 17 December 2009/Accepted: 13 July 2010/Published online: 26 August 2010

(C) SIMI 2010

\section{Introduction}

Foster Kennedy syndrome, named after neurologist Robert Foster Kennedy (1884-1952), describes unilateral ipsilateral optic atrophy and contralateral papilledema from an intracranial mass. This syndrome is unreliably associated with anosmia and ipsilateral proptosis [1]. It originates from variety of intracranial pathologies, but most often a subfrontal mass. We present a case of Foster Kennedy syndrome and review its etiology, pathology and incidence in intracranial tumors.

\section{Case report}

A 47-year-old woman was brought in by her family to the Emergency Department (ED) for psychiatric care. The patient had been homeless for 17 years prior to the visit, but had recently been taken in by her family, and brought in for evaluation of her personality changes. She denied any recent

\footnotetext{
S. Lotfipour · T. Bey $\cdot$ S. Rudkin

Irvine School of Medicine, University of California,

Orange, USA

K. Chiles

Highland General Hospital, Oakland, USA

J. A. Kahn

Thomas Jefferson University, Philadelphia, USA

S. Lotfipour $(\bowtie)$

Department of Emergency Medicine and Public Health, Clinical Science Education, University of California, Irvine School of Medicine, 200 S. Manchester Avenue, Suite 710, Orange, CA 92868, USA

e-mail: SHL@uci.edu
}

head trauma. She admitted to abusing crack cocaine for 13 years with her last use 4 months ago. She denied any trouble with ambulation, dizziness, and changes in hearing or other alterations in sensation. She denied any suicidal or homicidal ideation. The patient denied any auditory hallucinations, but did report that she had been experiencing visual hallucinations and visual disturbances for at least 6-8 months. She reported complete blindness in the left eye, and shadow perception in her right for an unknown length of time. Her past medical history was notable for major depression. The patient did not have a previous history of hallucinations or psychosis, and had never been hospitalized for psychiatric reasons. The patient was not on any medications, and was allergic to penicillin and codeine. The gynecologic, surgical and family histories were noncontributory.

Initial vital signs were: temperature $36.9^{\circ} \mathrm{C}$, pulse 86 beats/min, blood pressure $95 / 63 \mathrm{~mm} / \mathrm{Hg}$, respiratory rate 16 breaths/min, and oxygen saturation $100 \%$ on room air. On physical examination, the patient was alert and oriented to person, place and time. Her left pupil was nonreactive to direct light with a normal consensual light response and inability to count fingers with either eye. Extraocular movements were intact and cranial nerves IV through XII were normal. Further evaluation revealed she had anosmia, left optic nerve atrophy and right papilledema.

Laboratory studies were normal and urine toxicology was negative. Per hospital protocol initially a non-contrast computed tomography (CT) of the head revealed a large homogenous mass in the anterior cranial fossa (Fig. 1) measuring $8.0 \times 6.1 \mathrm{~cm}$. The mass had a calcified rim, and had eroded into the ethmoid sinus and bilateral orbits. A magnetic resonance imaging (MRI) confirmed the diagnosis of a subfrontal meningioma (Fig. 2).

The patient was started on phenytoin for seizure prophylaxis and dexamethasone to reduce cerebral edema. She 

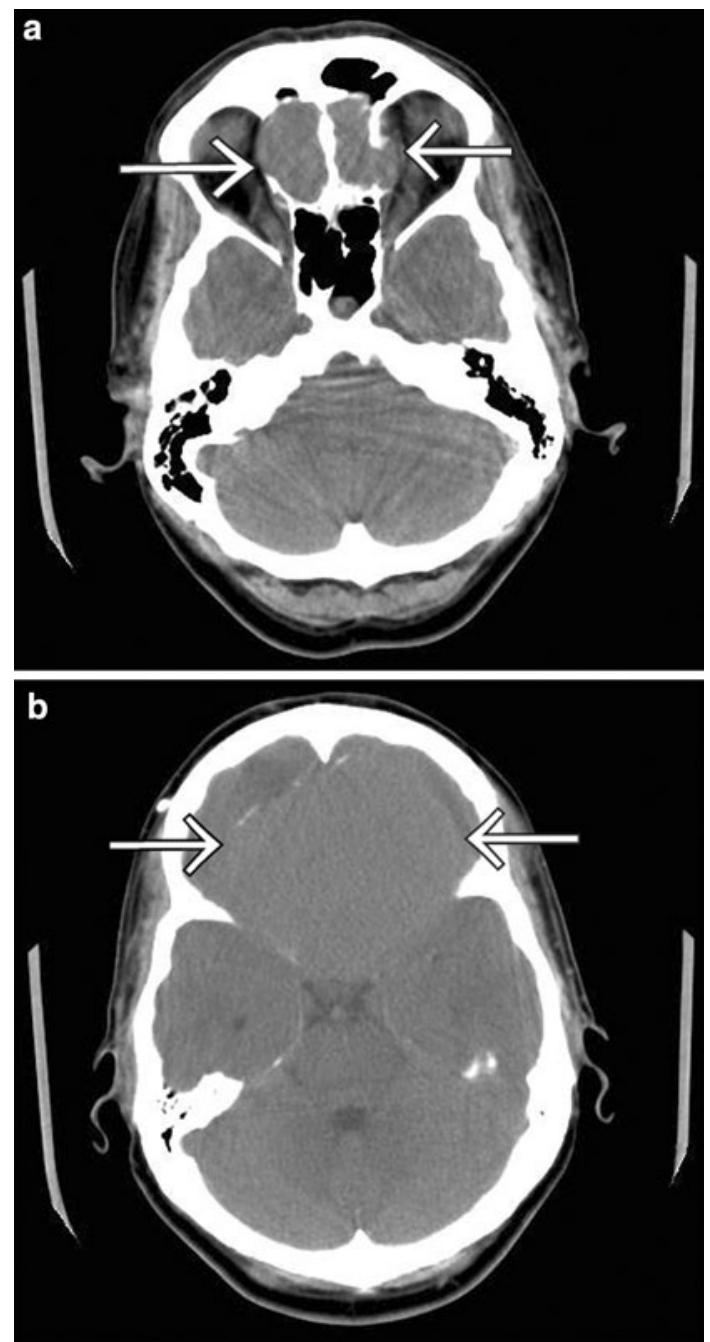

Fig. $1 \mathrm{CT}$ of the head without contrast shows a large anterior cranial mass with erosion into the orbits and ethmoid sinuses bilaterally (a) measuring $6.1 \times 8.0 \mathrm{~cm}(\mathbf{b})$

was discharged on hospital day 7 and scheduled for surgical resection following reduction in cerebral edema. The patient failed to return, and has since been lost to follow up.

\section{Discussion}

Foster Kennedy syndrome is uncommon, and numerous case series show its incidence to be less than $1 \%$ in conjunction with intracranial neoplasms. The largest series, performed in Germany by Tonnis in 1962 found 28 cases of Foster Kennedy syndrome in a series of 3,033 patients with intracranial tumors.

Most reported cases of Foster Kennedy syndrome are associated with an intracranial mass. The majority are caused by meningiomas of the olfactory groove, falx, sphenoidal wing or subfrontal regions, but cases have been
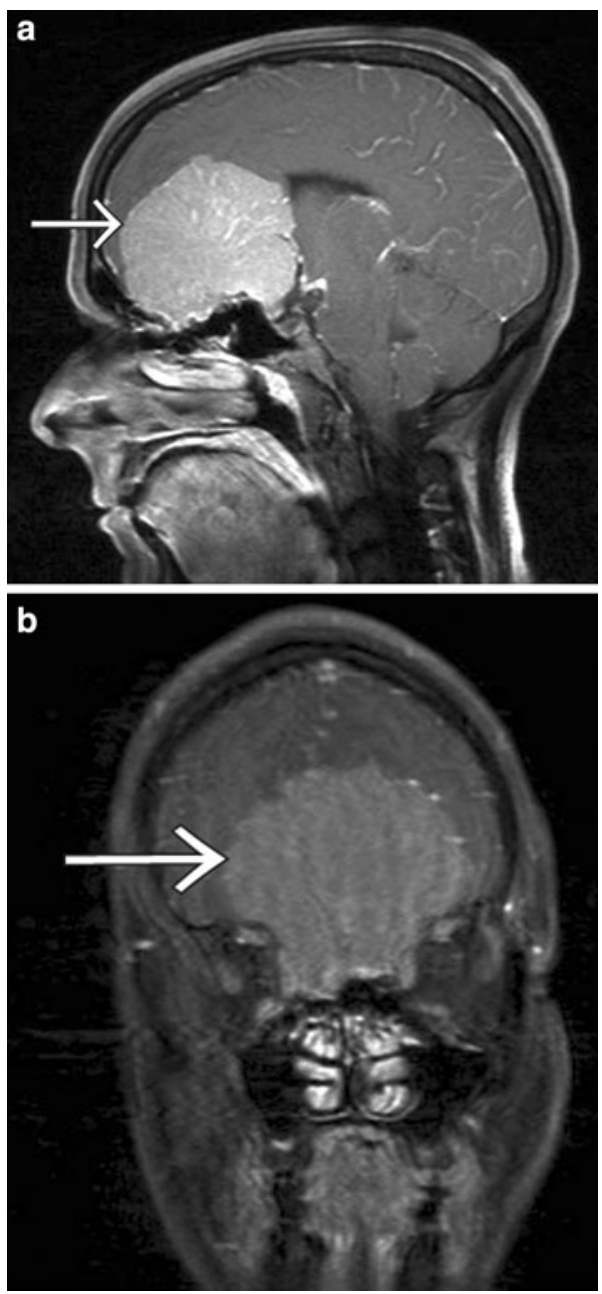

Fig. 2 MRI of the head shows a subfrontal meningioma with compression of the optic and olfactory tracts bilaterally $(\mathbf{a}, \mathbf{b})$

reported with mass lesions from frontal lobe abscesses, craniopharyngioma, pituitary adenoma, plasmacytoma, nasopharyngeal angiofibroma, neuroblastoma and aneurysms. Individuals with optic atrophy and contralateral papilledema without an intracranial mass have pseudoFoster Kennedy syndrome [2]. From 1909 to 2008, 4 of the 47 fully reported cases of Foster Kennedy syndrome occurred without an intracranial mass. These four cases were ascribed to congenital optic nerve hypoplasia, pachymeningitis, and two cases of anterior ischemic optic neuropathy in addition to an intracranial mass not located in the anterior cranial fossa $[2,3]$.

The mechanism of optic atrophy and contralateral papilledema has not been well elucidated. In 1911, Robert Foster Kennedy postulated that optic atrophy was a result of direct optic nerve compression and contralateral disc edema resulted from elevated intracranial pressure (ICP). A subsequent study supported this hypothesis in only $22 \%$ of the cases of Foster Kennedy syndrome [4]. Other possible 
mechanisms for the syndrome include bilateral optic nerve compression without elevated ICP and chronic elevated ICP without compression.

In this case there is clear evidence of bilateral optic nerve compression. Of the cases reviewed, this mechanism is most common, representing $33 \%$ of all fully documented cases [4]. Anosmia, an inconsistent finding, was present, and was caused by compression of the olfactory tract. Ipsilateral proptosis, which is rarely seen, was not seen despite the tumor's invasion into the orbits.

Imaging is useful in making the diagnosis. Although MRI is the imaging test of choice to evaluate suspected intracranial tumors, meningiomas are seen well with both computed tomography (CT scan) using contrast and MRI. The CT scan shows any associated bony hyperostosis while MRI is superior at soft tissue tumors. When using a CT scan, utilize thin slices $(1.5-3 \mathrm{~mm})$ in the suspected region. Bony involvement is important to determine, as it is the major contributor to recurrence [5].

Initial treatment for symptomatic brain tumors include corticosteroids, which may reduce peritumoral edema and decrease ICP. Anticonvulsants are given to all patients who have experienced a seizure. Since seizures are not only motor disturbances, thought should also be given to the location of the mass and potential epileptogenic effects. Given the frontal nature of this patient's tumor it is possible that the acute disturbances and psychiatric symptoms were seizure related, and she was started on anticonvulsants. However, most patients with brain tumors do not have a seizure as the first symptom. Randomized controlled trials have documented that prophylactic anticonvulsants in brain tumor patients do not protect these patients against future seizures, and may have substantial toxicity. Therefore, brain tumor patients who have not had a seizure should not be given prophylactic anticonvulsants [4].

\section{Conclusion}

Emergency physicians should consider Foster Kennedy syndrome in patients presenting for psychiatric conditions with changes in vision. Given this patient's initial presentation, an appropriate workup would include psychiatric and ophthalmologic consultation and a head CT scan, with and without contrast, but once diagnosed, the physician should obtain a neurosurgery consultation and an MRI study.

Acknowledgments Dr. Fong Tsai at the University of California, Irvine, for his help in evaluating the MRI images, Dr. Lynduh Soldavini for reviewing the manuscript and to Mr. Suleman Ahmed for his assistance with the images.

Conflict of interest None.

\section{References}

1. Stone JL, Vilensky J, McCauley, Tobi S (2005) Neurosurgery 100 years ago: the Queen Square letters of Foster Kennedy. Neurosurgery 57(5):797-808

2. Bansal S, Dabbs T, Long V (2008) Pseudo-Foster Kennedy syndrome due to unilateral optic nerve hypoplasia: a case report. J Med Case Rep 18:2-86

3. Limaye SR, Adler J (1990) Pseudo-Foster Kennedy syndrome in a patient with anterior ischemic optic neuropathy and a nonbasal glioma. J Clin Neuroophthalmol 10(3):188-192

4. DeAngelis LM (2005) General considerations. In: Rowland LP (ed) Merritt's neurology. Lippincott Williams \& Wilkins, USA

5. Zachariah SB, Khoromi S (2009) Meningioma, sphenoid wing: differential diagnoses and workup. eMedicine. Accessed 31 October 2009. http://www.emedicine.medscape.com/article/1215752diagnosis 